\title{
Outcomes and Predictors of 28-Day Mortality in Patients With Hematologic Malignancies and Septic Shock Defined by Sepsis-3 Criteria
}

Nirmala K. Manjappachar, MD¹; John A. Cuenca, MD¹; Claudia M. Ramírez, MD¹; Mike Hernandez, MS²; Peyton Martin, BS ${ }^{1}$;

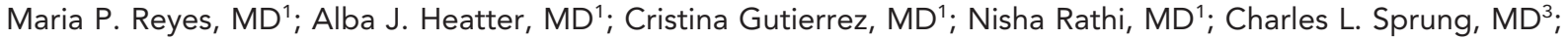

Kristen J. Price, $\mathrm{MD}^{1}$; and Joseph L. Nates, MD, MBA, CMQ, MCCM ${ }^{1}$

\begin{abstract}
Background: To describe short-term outcomes and independent predictors of 28-dayx mortality in adult patients with hematologic malignancies and septic shock defined by the new Third International Consensus Definitions (Sepsis-3) criteria. Methods: We performed a retrospective cohort study of patients admitted to the medical ICU with septic shock from April 2016 to March 2019. Demographic and clinical features and short-term outcomes were collected. We used descriptive statistics to summarize patient characteristics, logistic regression to identify predictors of 28-day mortality, and KaplanMeier plots to assess survival. Results: Among the 459 hematologic patients with septic shock admitted to the ICU, 109 (23.7\%) had received hematopoietic stem cell transplant. The median age was 63 years (range, 18-89 years), and 179 (39\%) were women. Nonsurvivors had a higher Charlson comorbidity index $(P=.007)$, longer length of stay before ICU admission $(P=.01)$, and greater illness severity at diagnosis and throughout the hospital course $(P<.001)$. The mortality rate at 28 days was $67.8 \%$ and increased with increasing sequential organ failure assessment score on admission (odds ratio [OR], 1.11; $95 \% \mathrm{Cl}$, 1.03-1.20), respiratory failure (OR, 3.12; 95\% Cl, 1.49-6.51), and maximum lactate level (OR, 1.16; $95 \% \mathrm{Cl}, 1.10-1.22)$. Aminoglycosides administration $(\mathrm{OR}, 0.42 ; 95 \% \mathrm{Cl}, 0.26-0.69)$, serum albumin $(\mathrm{OR}$, $0.51 ; 95 \% \mathrm{Cl}, 0.31-0.86)$, and granulocyte colony-stimulating factor (G-CSF) (OR, 0.40; 95\% Cl, 0.24-0.65) were associated with lower 28-day mortality. Life support limitations were present in $81.6 \%$ of patients at death. At 90 days, $19.4 \%$ of the patients were alive. Conclusions: Despite efforts to enhance survival, septic shock in patients with hematologic malignancies is still associated with high mortality rates and poor 90-day survival. These results demonstrate the need for an urgent call to action with higher awareness, including the further evaluation of interventions such as earlier ICU admission, aminoglycosides administration, and G-CSF treatment.
\end{abstract}

J Natl Compr Canc Netw 2022;20(1):45-53 doi: $10.6004 /$ jnccn.2021.7046

${ }^{1}$ Department of Critical Care, Division of Anesthesiology, Critical Care, and Pain, and ${ }^{2}$ Department of Biostatistics, The University of Texas MD Anderson Cancer Center, Houston, Texas; and ${ }^{3}$ Department of Anesthesiology, Critical Care Medicine and Pain Medicine, Hadassah Medical Center, Hebrew University of Jerusalem, Jerusalem, Israel.

\section{Background}

Cancer survival rates have improved significantly over the past 3 decades. ${ }^{1}$ As cancer mortality decreases and the cancer population grows older, the burden of cancer continues to increase. ${ }^{2,3}$ This progressively larger population of patients with cancer, especially those with hematologic malignancies, have a significantly higher risk of developing sepsis compared with the noncancer population. ${ }^{4,5}$ Recent epidemiologic reports indicate that 1 in 5 patients with sepsis has cancer ${ }^{6}$ and $8.5 \%$ of all cancer deaths are associated with severe sepsis. ${ }^{4}$

Between 2009 and 2014, sepsis incidence in the United States increased by $10.3 \%$ (95\% CI, $7.2 \%-13.3 \%) .{ }^{6}$ In 2017 , 11 million deaths related to sepsis were reported worldwide, comprising $19.7 \%$ of all deaths that year. ${ }^{7}$ In the United States, it is estimated that one-third of hospitalized patients' deaths are related to sepsis. ${ }^{6,8}$ Overall, the sepsis mortality rate ranges between $12.5 \%$ and $17 \% .{ }^{6,9,10}$ However, based on the severity of the sepsis, mortality can be as low as $5.6 \%$ in sepsis without organ dysfunction or can increase to $34.2 \%$ for septic shock. ${ }^{10}$ In a cohort of patients with solid tumors and hematologic malignancies from 1994 to 2015, those with sepsis and septic shock had a 30-day mortality of $42.6 \% .^{5}$

Most large epidemiologic studies report sepsis in combination with septic shock and do not address patients with cancer..$^{6-9}$ Some studies address critically ill patients with hematologic malignancies but not sepsis or septic shock, ${ }^{11,12}$ whereas other reports that addressed septic shock in this population predated the latest 2016 international consensus definitions for sepsis and septic shock and thus used Sepsis-2 definitions. ${ }^{13,14}$ Some studies included small cohorts or were spread over long periods, leading to time bias. ${ }^{5,15,16}$ In other studies, severe sepsis and septic shock are reported together. ${ }^{5,14}$ This article reports on the short-term outcomes and the independent predictors of 28-day mortality in adult patients with

\section{See JNCCN.org for supplemental online content.}


hematologic malignancies and septic shock using the new Third International Consensus Definitions for Sepsis and Septic Shock (Sepsis-3) clinical criteria in a high-volume comprehensive cancer center. ${ }^{17}$ We hypothesized that the mortality rates of septic shock in patients with hematologic malignancies remain unacceptably high.

\section{Methods}

\section{Design, Setting, and Participants}

This retrospective longitudinal study was conducted with the approval of The University of Texas MD Anderson Institutional Review Board. The hospital ICU is a 52-bed facility, with a well-delineated and open admission policy, as previously described. ${ }^{18,19}$ Other features include an ICU admission triage service, a rapid response system, an outreach program, multidisciplinary rounds, evidence-based protocols, and a historical average of 3,500 annual admissions. ${ }^{19}$ The study population was identified from all adult patients with cancer (age $\geq 18$ years) admitted to the medical ICU from April 1, 2016, to March 31, 2019, who had an ICD-10 diagnosis code for sepsis or diagnostic-related group (DRG 870, 871, or 872) as described by Paoli et al. ${ }^{9}$

To this population, we applied inclusion criteria of having a diagnosis of hematologic malignancy and septic shock meeting Sepsis-3 criteria (suspected infection, persistent hypotension requiring vasopressors to maintain a mean arterial pressure of $\geq 65 \mathrm{~mm} \mathrm{Hg}$ despite adequate volume resuscitation, and a serum lactate level of $>2 \mathrm{mmol} / \mathrm{L}){ }^{13}$ Patients with a lactate level of $\leq 2 \mathrm{mmol} / \mathrm{L}$ after resuscitation prior to ICU admission, those without septic shock, and those with sepsis concomitant with other types of shock, such as cardiogenic, hemorrhagic, or neurologic shock, were excluded to reduce bias. We also excluded all postsurgical patients.

\section{Data Sources and Measurements}

Demographic and clinical information such as age, sex, comorbidities, Charlson comorbidity index (CCI), and characteristics of cancer, such as cancer diagnosis, histologic type, and current status (remission, relapse, or progression), were extracted from patients' electronic medical records. We also collected relevant information on cancer therapy, including the recent history of chemotherapy and characteristics of stem cell transplants (SCTs). We determined the source of admission to the ICU (emergency department or hospital ward), the main reason for ICU admission, presence of sepsis on admission, the sequential organ failure assessment (SOFA) score at the time of septic shock diagnosis, maximum SOFA score during the ICU stay, length of stay (pre-ICU, ICU, hospital), neutropenia (absolute neutrophil count $[\mathrm{ANC}] \leq 1,500$ / $\mathrm{mm}^{3}$ ), severe neutropenia (ANC $<500 / \mathrm{mm}^{3}$ ), therapeutic interventions during the ICU stay (use of vasopressors, mechanical ventilation, dialysis, chemotherapy), use of noninvasive mechanical ventilation, renal replacement therapy, and additional laboratory values. Outcomes included 28-day mortality, ICU mortality, hospital mortality, 90-day mortality, and ICU resource utilization such as ICU length of stay, days on ventilator, and days on vasopressors. The change in code status during ICU stay (donot-resuscitate [DNR] order), life support limitations or withdrawal, survival status at the time of hospital discharge, and the discharge destination were captured up to 90 days after ICU admission. We followed the Strengthening the Reporting of Observational Studies in Epidemiology (STROBE) guidelines. ${ }^{20}$

\section{Statistical Analysis}

Descriptive statistics were used to summarize the demographic, clinical, and treatment-related patient features. Bivariate analyses were conducted to explore associations with 28-day ICU mortality for all candidate predictors. Predictors were screened using logistic regression in a univariable setting. Continuous predictors were assessed for linearity in functional form. All potential predictors whose relationship with 28-day mortality met a threshold of $P<.10$ were considered for full model inclusion. After we established a full model of all candidate predictors, backward elimination was used to isolate independent predictors where the probability of covariate retention was $P<.05$. Odds ratio (OR) estimates and $95 \%$ confidence intervals summarized study findings. The multivariable logistic regression model was critically assessed using the Hosmer-Lemeshow goodness-of-fit test. ${ }^{21}$ Also calculated was the concordance index, which specifies the area under the receiver operating characteristic curve. ${ }^{22}$ To assess model discrimination visually, probability estimates of 28-day mortality emanating from the multivariable model used terciles to categorize patients as low, moderate, and high risk for early mortality after ICU admission. Survival was computed from the date of ICU admission to the patient's date of death or date of the last follow-up, at which time patients were censored. Kaplan-Meier plots were generated to assess survival distributions based on patient characteristics of interest. Survival was artificially censored at 90 days because of the high rate of mortality experienced at day 28. $P$ values of $<.05$ were considered statistically significant. Statistical analyses were conducted using STATA, version 15 (StataCorp LP).

\section{Results}

During the 3-year study period, 86,231 patients were admitted, of whom $6,486(7.5 \%)$ were admitted to the ICU. Among them, 2,242 (34.6\%) had sepsis, and of those, 809 (36\%) met Sepsis-3 criteria for septic shock (Figure 1). Of these patients with septic shock, 459 also had hematologic 


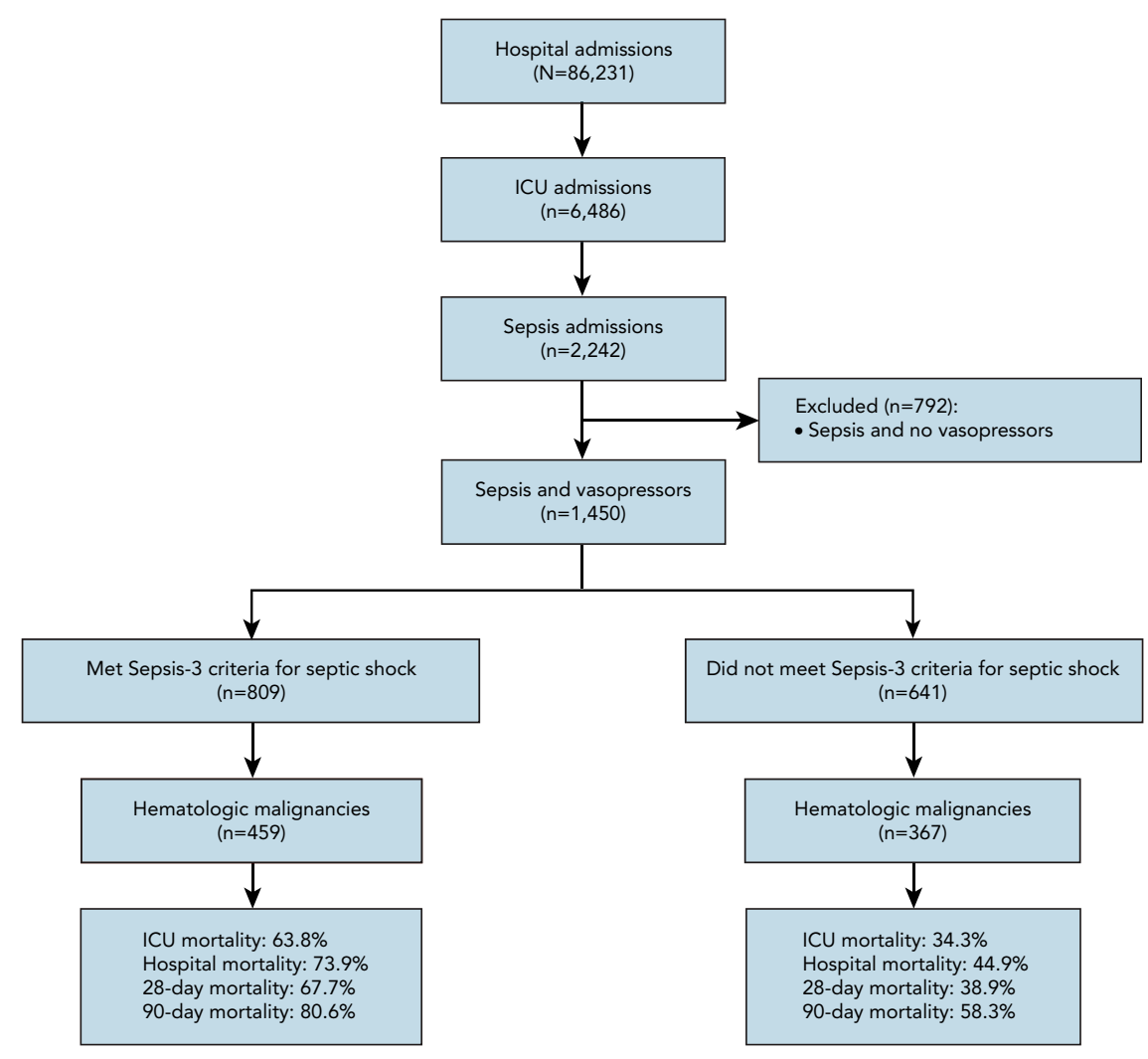

Figure 1. Flowchart of included participants by the Sepsis-3 definition.

malignancies: 284 (61.9\%) had leukemia, 97 (21.1\%) had lymphoma, and 78 (17\%) had other hematologic malignancies (supplemental eFigure 1, available with this article at JNCCN.org). By day 28 after ICU admission, 311 patients $(67.7 \%)$ died and 148 (32.2\%) survived. Of the nonsurvivors, 147 (47.2\%) had relapsed/refractory disease at ICU admission. A total of 109 patients (23.7\%) had a history of SCT, $20 \%$ autologous and $80 \%$ allogeneic, with no mortality difference between the transplant types at 28 days $(P=.165)$; nor was there a difference in 28-day mortality rate between patients with and without a SCT $(67 \%$ [73/109] vs $68 \%$ [238/350], respectively; $P=.83$ ). There were more men (61\%) than women, and most patients were White (61.4\%). The median age was 63 years (range, 18-89 years), and the median body mass index was $27 \mathrm{~kg} / \mathrm{m}^{2}$ (range, $15-57 \mathrm{~kg} / \mathrm{m}^{2}$ ). The primary source of admission was the hospital ward (71.4\%), 35 patients (7.6\%) had pre-ICU cardiac arrests, and $7.6 \%$ were later readmitted to the ICU. Approximately half of the patients had $<3$ comorbidities (51.6\%), and one-third had a history of diabetes (31\%). The demographic characteristics according to 28-day survival status are summarized in Table 1 , and comorbidities are summarized in supplemental eTable 1.

Respiratory failure was present during the ICU stay in $88.8 \%$ of patients, and of those, $293(71.8 \%)$ died $(P<.001)$.
Of the 277 patients who required intubation, $174(62.8 \%)$ were intubated within 24 hours of admission, and 199 (71.8\%) died, whereas among the 45 patients who required only high-flow oxygen or noninvasive ventilation, 26 (57.8\%) died $(P=.011)$.

Characteristics and outcomes are compared between survivors and nonsurvivors in Tables 1 and 2. Patients who died within 28 days had significantly higher lactate levels at the time of septic shock diagnosis (mean [SD], 7.8 [6.4] vs 4.7 [3.0] $\mathrm{mmol} / \mathrm{L}$ ) and at ICU admission (mean [SD], 10 [6.9] vs 5.7 [3.9] mmol/L; $P<.0001)$ but significantly lower serum albumin levels (mean [SD], 2.5 [0.5] vs $2.6[0.5] \mathrm{g} / \mathrm{dL} ; P<.0001$ ) (supplemental eTable 2). All the patients were treated with at least one antibiotic. Most patients received antifungals (81.2\%), followed in frequency by antivirals (61.4\%) and aminoglycosides (60. $7 \%$ ). Microbial organisms were isolated in $66.6 \%$ of patients (supplemental eTable 3). Among them, the patients with fungal isolates had the highest mortality rate $(72.7 \%)$. Mortality was significantly lower for patients with gram-negative organisms compared with the remainder of the cohort ( $61 \%$ vs $71 \% ; P=.029$ ), and in patients who received meropenem, aminoglycosides, or cefepime $(P<.0001)$, as well as those who received hydrocortisone $(P=.044)$ (supplemental eTable 4 ). 


\section{Table 1. Study Cohort Characteristics}

\section{Variable}

Total, $n$

Age, median (range), y

Female

Race/Ethnicity

White

African American

Hispanic

Asian American

Other $^{\mathrm{a}}$

$\mathrm{BMI}$, median (range), $\mathrm{kg} / \mathrm{m}^{2}$

Underlying malignancy

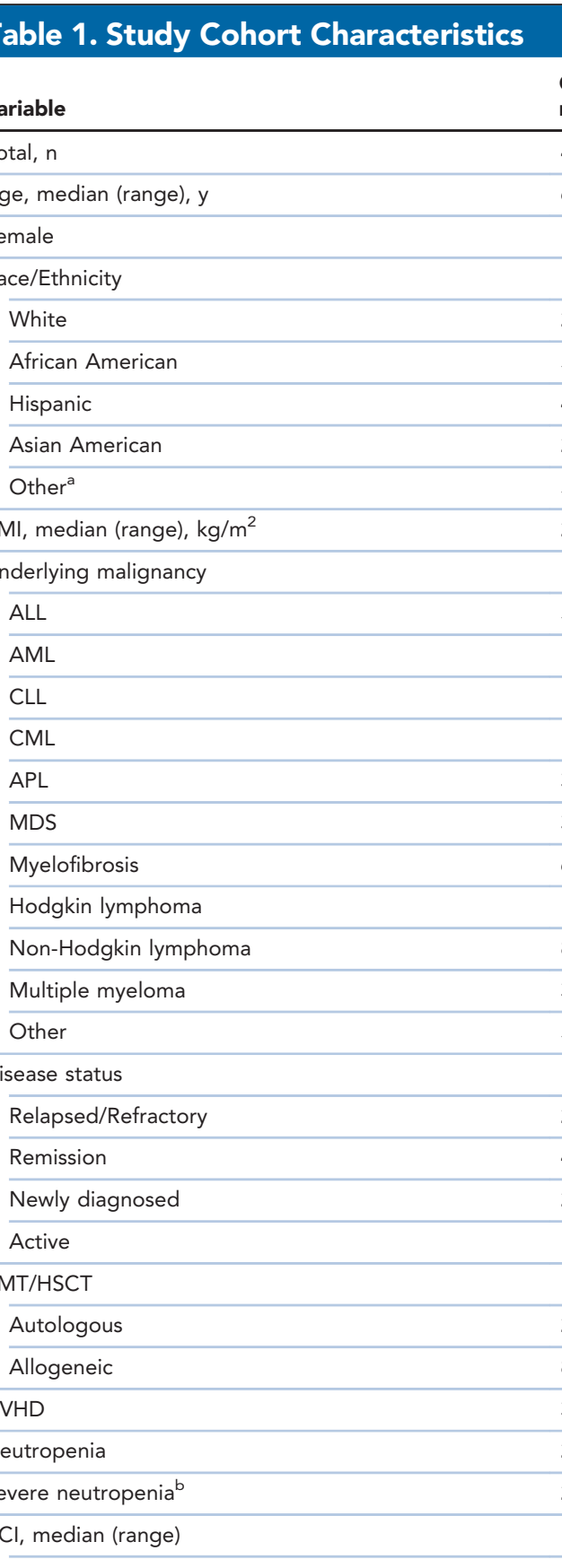

CLL

$\mathrm{CML}$

APL

MDS

Myelofibrosis

Hodgkin lymphoma

Non-Hodgkin lymphoma

Multiple myeloma

Other

Disease status

Relapsed/Refractory

Remission

Newly diagnosed

Active

BMT/HSCT

\section{Autologous}

Allogeneic

GVHD

Neutropenia

Severe neutropenia ${ }^{b}$

$\mathrm{CCl}$, median (range)

\section{Overall}

n (\%)

459

63 (18-89)

179 (39.0)

$282(61.4)$

57 (12.4)

41 (8.9)

$25(5.4)$

54 (11.7)

27 (15-57)

54 (11.7)

$198(43.1)$

17 (3.7)

$12(2.6)$

$3(0.6)$

$30(6.5)$

$6(1.3)$

$10(2.1)$

87 (18.9)

37 (8)

5 (1)

$217(47.2)$

43 (9.3)

$20(4.3)$

177 (38.5)

109 (23.7)

22 (4.7)

87 (18.9)

$33(7.1)$

298 (64.9)

$247(53.8)$

$5(1-18)$

10-y survival estimate

ECOG performance status

\begin{tabular}{ll}
\hline 0 & $55(11.9)$ \\
\hline 1 & $163(35.5)$ \\
\hline 3 & $97(21.1)$ \\
\hline 4 & $90(19.6)$ \\
\hline Information not available $^{c}$ & $36(7.8)$ \\
\hline
\end{tabular}

21 (0-96)
Survivors

n (\%)

148

60 (19-84)

61 (41.2)

79 (53.3)

18 (12.1)

17 (11.4)

$8(5.4)$

26 (17.5)

27 (15-50)

$26(17.5)$

47 (31.7)

7 (4.7)

4 (2.7)

2 (1.3)

7 (4.7)

$1(0.6)$

6 (4)

29 (19.5)

$16(10.8)$

3 (2)

70 (47.2)

21 (14.1)

5 (3.3)

51 (34.4)

36 (24.3)

$10(6.7)$

26 (17.5)

$10(6.7)$

93 (62.8)

83 (56.5)

4 (1-11)

37 (0-96)

19 (12.8)

69 (46.6)

28 (18.9)

19 (12.8)

7 (4.7)

6
5 (2-18)

.007

$21(0-90)$

.018

.003

36 (11.5)

.076

147 (47.2)

$22(7)$

$126(40.5)$

$73(23.4)$

$12(3.8)$

.841

.165

61 (19.6)

$23(7.3)$

.806

205 (65.9)

.49

.52

94 (30.2)

$69(22.1)$

71 (22.8)

29 (9.3)

12
.002

.501

.038

.007

98

Value

7




\begin{tabular}{|c|c|c|c|c|}
\hline Emergency & $131(28.5)$ & $58(39.1)$ & $73(23.4)$ & \\
\hline Hospital ward & $328(71.4)$ & $90(60.8)$ & $238(76.5)$ & \\
\hline Other $^{d}$ & $136(29.6)$ & $31(20.9)$ & $105(33.7)$ & \\
\hline CPR before ICU admission & $35(7.6)$ & $8(5.4)$ & $27(8.6)$ & .216 \\
\hline SOFA at SS diagnosis, median (range) & $11(4-21)$ & $10(4-18)$ & $12(4-21)$ & $<.001$ \\
\hline Maximum SOFA, median (range) & $14(4-22)$ & $12(4-22)$ & $14(6-21)$ & $<.001$ \\
\hline Recent chemotherapy & $65(14.1)$ & $24(16.2)$ & $41(13.1)$ & .313 \\
\hline Checkpoint inhibitors & $11(2.3)$ & $2(1.3)$ & $9(2.8)$ & .312 \\
\hline
\end{tabular}

Abbreviations: $A L L$, acute lymphocytic leukemia; $A M L$, acute myeloid leukemia; $A P L$, acute promyelocytic leukemia; $B M I$, body mass index; $B M T$, bone marrow transplant; CCl, Charlson comorbidity index; CLL, chronic lymphocytic leukemia; CML, chronic myeloid leukemia; CPR, cardiopulmonary resuscitation; GVHD, graftversus-host disease; HSCT, hematopoietic stem cell transplant; MDS, myelodysplastic syndromes; SOFA, sequential organ failure assessment score; SS, septic shock. "One patient with missing race/ethnicity was classified as "other."

${ }^{\mathrm{b}}$ Absolute neutrophil count $<500 / \mathrm{mm}^{3}$.

${ }^{\mathrm{C}}$ Patients with unavailable information were not included for statistical comparison.

IIncludes respiratory failure; cardiac, neurologic, renal, multiorgan causes; coagulopathy.

ICU mortality was $63.8 \%$, 28-day mortality was $67.7 \%$, hospital mortality was $73.9 \%$, and the 90 -day mortality was $80.6 \%$. Patients who had an allogeneic SCT with graft-versus-host disease had the lowest survival rates at 90 days (4\%). A subanalysis of septic shock patients not meeting Sepsis-3 criteria demonstrated significantly lower ICU (34.3\%; $P<.0001)$, 28-day (38.9\%; $P<.0001)$, hospital (44.9\%; $P<.0001)$, and 90-day (58.3\%; $P<.0001$ ) mortality rates than patients meeting Sepsis-3 criteria (Figure 1; supplemental eTable 5). Two-thirds of patients (67.5\%) had life supportive measures withheld (DNR) after ICU admission. At 90 days, 370 patients $(80.6 \%)$ had died. Of those patients, $302(81.6 \%)$ had a DNR order in place and $209(56.4 \%)$ had life support withdrawn at the time of death (supplemental eTable 6). The 1-year survival of the Sepsis-3 cohort was $10 \%(\mathrm{n}=46)$. The patient distribution by SOFA score and survival at 28 days is shown in supplemental eFigure 2.

Kaplan-Meier plots were used to visually assess survival distributions of patients by hematologic malignancy, leukemia subtype, SCT type, and severe neutropenic status at 90 days (Figure 2). The reduced logistic regression model derived using backward elimination resulted in a 6-covariate model containing: admission SOFA score, respiratory failure, aminoglycoside use, highest lactate level, serum albumin level, and G-CSF use. Multivariable analysis showed that higher serum albumin level (OR, 0.51; 95\% CI, 0.31-0.86; $P=.011$ ), G-CSF (OR, 0.4; 95\% CI, 0.24-0.65; $P<.001$ ), and therapy with aminoglycosides (OR, $0.42 ; 95 \%$
CI, $0.26-0.69 ; P=.001)$ were associated with lower mortality, whereas respiratory failure (OR, 3.12; 95\% CI, 1.49-6.51; $P=.003$ ), higher lactate level (OR, 1.16; 95\% CI, 1.10-1.22; $P<.001$ ), and higher admission SOFA score (OR, 1.11; 95\% CI, 1.03-1.20; $P=.005$ ) were associated with higher mortality (Figure 3). There was no mortality difference between patients with severe versus nonsevere neutropenic septic shock $(P=.36)$ (supplemental eTable 7). Patients who received G-CSF had a lower hospital and 28-day mortality, but the difference disappeared at 90 days (supplemental eTable 8). The Hosmer-Lemeshow goodness-of-fit test suggested adequate model fit (chi-square, 7.03; $d f=8$; $P=.533$ ). The prediction model discrimination is visually represented in supplemental eFigure 3 . The $\mathrm{C}$-index associated with this clinical prediction model was 0.795 , suggesting moderate to good discrimination. ${ }^{21,22}$

\section{Discussion}

In this study of a large cohort of adult patients with hematologic malignancies and septic shock by Sepsis3 criteria, two-thirds of the patients died within 28 days, and only 1 in 5 were alive at 90 days. Multivariable analysis showed a survival benefit among those receiving aminoglycosides, those given G-CSF, and those with higher albumin levels, whereas respiratory failure, higher lactic acid levels, and higher SOFA scores were associated with increased mortality. The highest mortality was in transplant patients who had graft-versus-host disease. 


\section{Table 2. Resource Utilization}

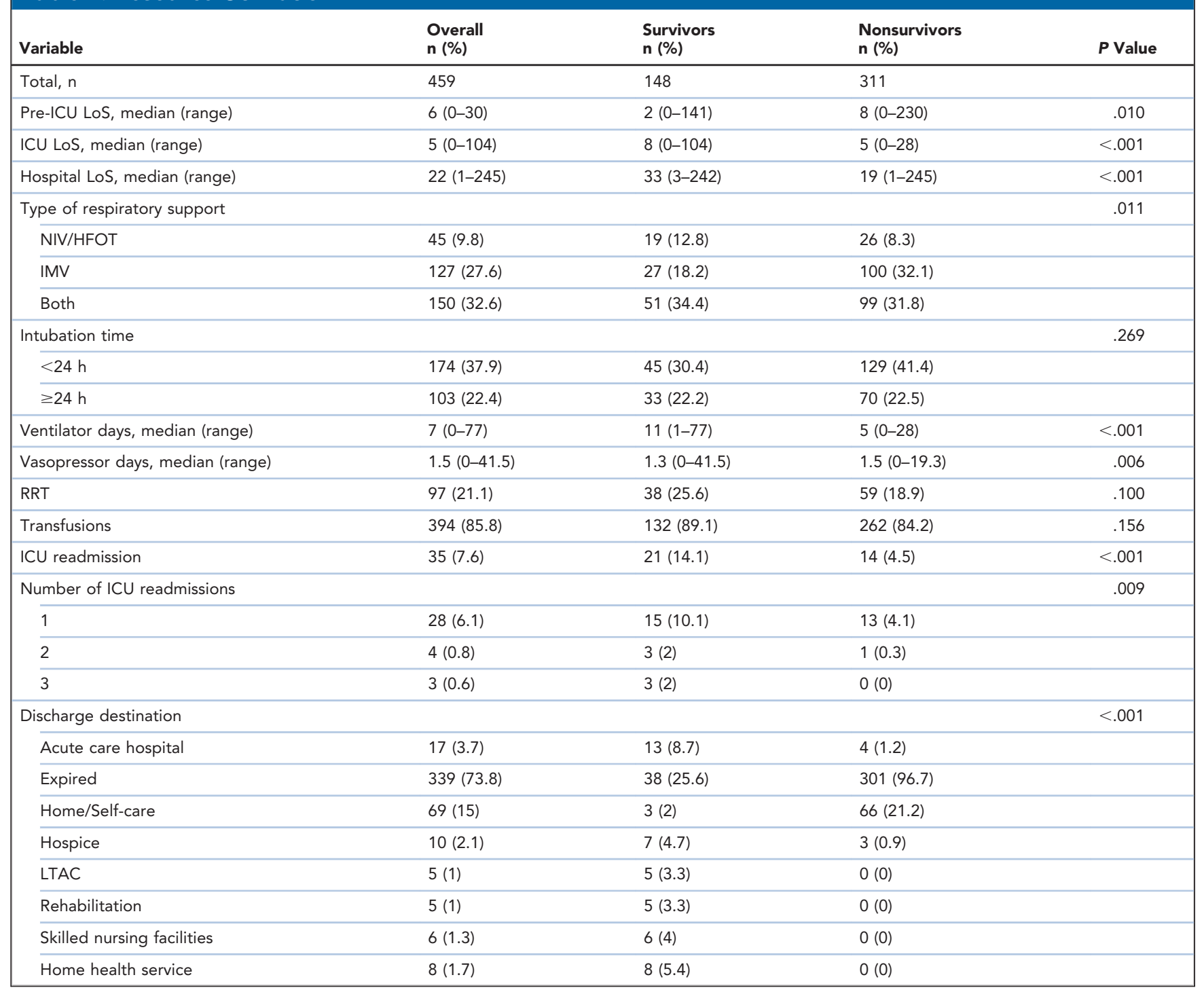

Patients with unavailable information were not included for statistical comparison.

Abbreviations: HFOT, high-flow oxygen therapy; ICU, intensive care unit; IMV, invasive mechanical ventilation; LoS, length of stay; LTAC, long-term acute care facility; NIV, noninvasive ventilation; RRT, renal replacement therapy.

Life support limitations were present in 8 of every 10 patients at the time of death.

The survival of patients with cancer ${ }^{1,19}$ and of those with sepsis has improved during the past 2 decades, especially after outstanding international efforts in sepsis education and development of therapeutic strategies to reduce sepsis mortality ${ }^{23}$; nevertheless, this study demonstrates poor outcomes in patients with hematologic malignancies meeting Sepsis-3 criteria. This cohort included a population with high SOFA and CCI scores, high need for ventilatory support, and high incidence of acute kidney injury. In contrast, patients with septic shock not meeting Sepsis-3 criteria demonstrated high but significantly lower mortality. These differences highlight the impact of the Sepsis-3 definition. ${ }^{24,25}$ This correlation has been described previously. ${ }^{26,27}$ In a cohort of 241 patients with solid tumors and 112 with hematologic malignancies, Nathan et $\mathrm{al}^{28}$ compared hospital mortality from septic shock between the Sepsis-2 and Sepsis-3 definitions. Although the use of invasive mechanical ventilation in their cohort was only $19 \%$, they found a mortality of $60 \%$ with the Sepsis-2 definition in contrast to $68 \%$ with the Sepsis-3 definition.

Most large studies in this area analyze sepsis in general and do not detail the outcomes of septic shock in cancer independently. Williams et $\mathrm{al}^{4}$ showed an overall sepsis incidence of $4.9 \%$, with $2.4 \%$ needing the ICU in 1999 , similar to the $2.6 \%$ incidence of cancer hospitalizations experiencing sepsis and ICU admission we found in our 
A

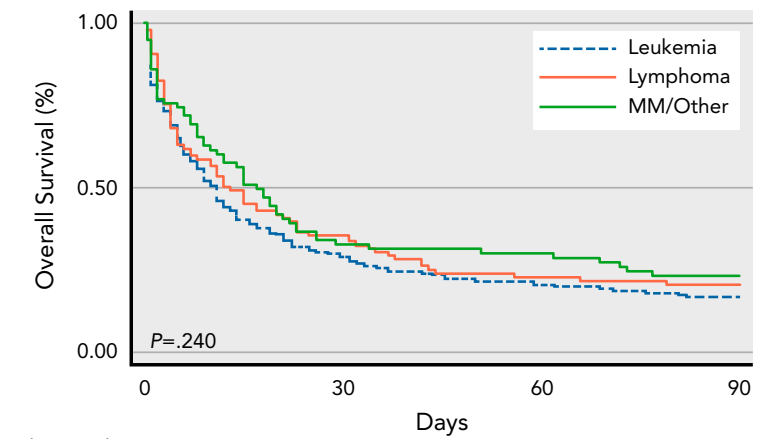

Number at risk

$\begin{array}{rr}\text { Leukemia } & 284 \\ \text { Lymphoma } & 97\end{array}$

MM/Other 78

C

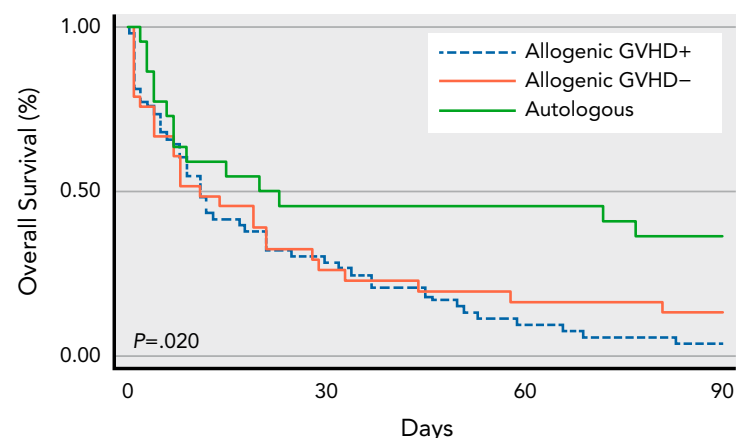

Number at risk

Allogenic GVHD+ 53

Allogenic GVHD- 33

Autologous 22
B

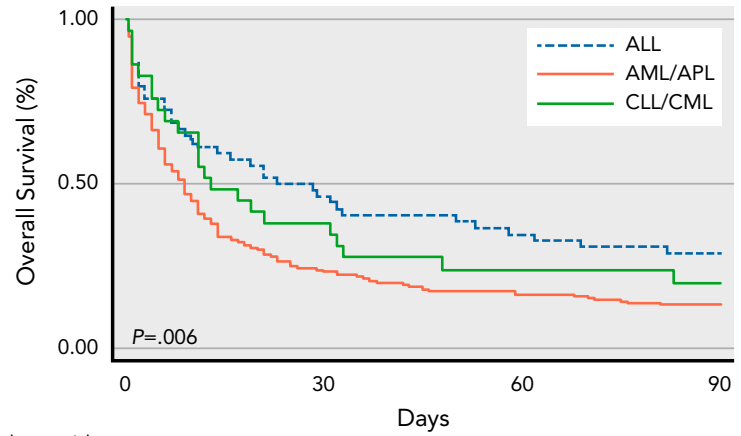

Number at risk

ALL 54

AML/APL 201

CLL/CML 29

D

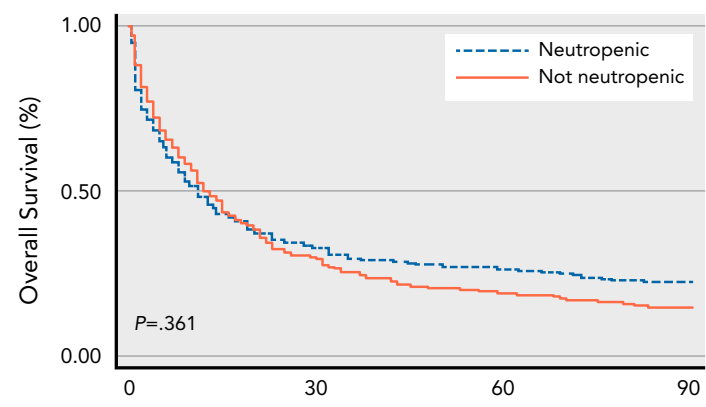

Number at risk

Neutropenic 247

Sot neutropenic 208
Days

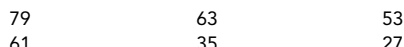

Figure 2. Survival curves by (A) hematologic malignancy type, (B) leukemia type, (C) hematopoietic stem cell transplant type, and (D) severe neutropenia status (absolute neutrophil count $<500 / \mathrm{mm}^{3}$ ). Differences between the curves were assessed using the log-rank test.

Abbreviations: ALL, acute lymphocytic leukemia; AML, acute myeloid leukemia; $A P L$, acute promyelocytic leukemia; $C L L$, chronic lymphocytic leukemia; CML, chronic myeloid leukemia; GVHD, graft-versus-host disease; MM, multiple myeloma.

study. Williams et $\mathrm{al}^{4}$ reported a $36.1 \%$ combined (severe sepsis/septic shock) mortality in patients with hematologic malignancies. Similarly, Cooper et $\mathrm{al}^{29}$ recently reported a cohort of 20,975 patient with and without cancer hospitalized with sepsis during a period of 12 years (2003-2014); of the 7,489 patients with cancer, 2,866 had hematologic malignancies (only $38.1 \%$ required vasopressors) and an overall sepsis in-hospital mortality rate of $28.8 \%$. Hensley et $\mathrm{al}^{30}$ reported $27.9 \%$ in-hospital mortality in patients with cancer with sepsis during 2013 to 2014, but no details about septic shock were provided. Lemiale et $\mathrm{al}^{5}$ reported a 30 -day mortality of $39.9 \%$ in a cohort of patients with sepsis/septic shock and cancer, $82 \%$ of whom had hematologic malignancies, over a period of 21 years (1994-2015); mortality of the patients with hematologic malignancies and septic shock was not reported.

The decrease in the reported historical sepsis mortality rates over the past 20 years can be misleading, and many practitioners may underestimate the prognosis of septic shock. An Australian study of ICU patients found $18.4 \%$ in-hospital mortality for those with sepsis and $22 \%$ for those with septic shock in $2012 .{ }^{31}$ In a recent systematic review (2005-2018) of the frequency and mortality of septic shock in Europe and North America, Vincent et $\mathrm{al}^{32}$ reported an ICU mortality of $37.3 \%$ (95\% CI, $31.5 \%-43.5 \%)$ and a hospital mortality of $39.0 \%(95 \%$ CI, $34.4 \%-43.9 \%$ ) in patients with septic shock defined using the Sepsis-2 criteria. When septic shock was defined using the Sepsis-3 criteria, the ICU mortality increased to $51.9 \%$ (95\% CI, $43.9 \%-59.8 \%)$ and the hospital mortality to $52.1 \%$ (95\% CI, $51.6 \%-52.6 \%$ ). Azoulay et $\mathrm{al}^{11}$ also reported a high hospital mortality in a large general cohort (including patients without sepsis or shock) of ICU patients with hematologic malignancies, of whom 51\% required vasopressors, $26 \%$ required renal replacement therapy, and $48 \%$ required invasive mechanical ventilation; mortality rates were $57.5 \%$, $59.2 \%$, and $60.5 \%$, respectively.

The increased risks of developing sepsis with organ dysfunction and the higher mortality of allogeneic SCT patients with graft-versus-host disease have been described..$^{33}$ Patients admitted to the ICU within 100 days of allogeneic transplant have a hospital mortality of $64 \%$ and a 1-year survival rate of $15 \% .{ }^{34}$ Therefore, given that 


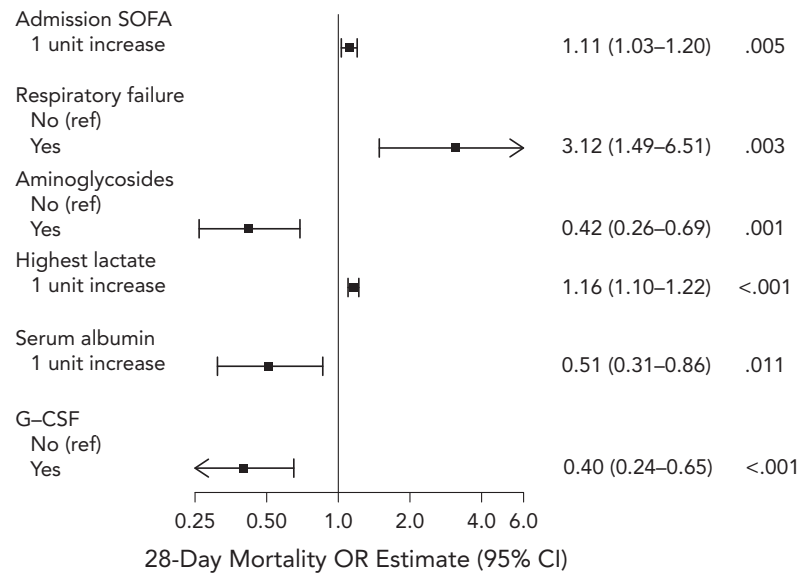

Figure 3. Multivariable analysis of risk factors associated with 28-day mortality $(n=456$; Hosmer-Lemeshow goodness-of-fit test, 7.03, $d f=8 ; P=.533 ; C$-index $=0.795)$.

Abbreviations: G-CSF, granulocyte colony-stimulating factor; $\mathrm{OR}$, odds ratio; SOFA, sequential organ failure assessment score.

$80 \%$ of transplants in our cohort were allogeneic, the low survival rate seen in our study is not surprising.

We also observed that patients with acute myeloid leukemia (AML) had a lower 90-day survival rate compared with those with other types of acute leukemias ( $12 \%$ vs $28 \%$ in acute lymphocytic leukemia) (Figure 2). In a cohort of 2,173,776 adults discharged from hospitals in Texas, those with AML had an increased incidence of sepsis ( $16 \%$ vs $4 \%)$ and higher sepsis-associated hospital mortality ( $30 \%$ vs $21 \%$ ) compared with patients with non-AML cancer. ${ }^{35}$ In addition, when patients with AML develop renal failure, their 8-week mortality is as high as $65 \%$ regardless of dialysis. ${ }^{36}$

Although our study showed no difference in mortality between patients with and without neutropenia, we did find that those who received G-CSF had a reduced risk of death at 28 days. A recent meta-analysis found an association between neutropenia and mortality, and this association disappeared after the analysis was adjusted for the use of G-CSF therapy. ${ }^{37}$ The association we observed between the use of aminoglycosides and improved outcomes has also been previously reported, thus giving strength to this association. ${ }^{38}$ The timing of antibiotics has also been proposed as a significant factor in the reduction of mortality among patients with sepsis. ${ }^{39,40}$ A recent publication of 162 ambulatory patients with cancer suspected of developing sepsis/septic shock treated with early fluid resuscitation and aggressive antibiotic treatment (meropenem, tobramycin, and linezolid) reported an overall mortality rate of $4 \% .{ }^{41}$

Our study was conducted in a high-volume, quaternary comprehensive cancer center specialized in treating all types of cancers and experimental therapies. Overall, our findings are consistent with the existing literature. At the same time, it is a retrospective cohort and single-center study, which may limit the generalizability of the results. However, we implemented strategies to diminish selection bias, such as data validation by an ICU physician. Another limitation is that we excluded patients with sepsis and a concomitant cardiogenic shock. Despite efforts to validate our findings, there is a possibility that a fraction of the patients with septic shock and sepsis-induced cardiomyopathy could have been omitted. Additionally, given our institution's nature, we manage large volumes of patients with advanced cancer, which may have skewed the patient population toward higher percentages of multiorgan dysfunction/failure and less organ reserve to respond during critical illness.

\section{Conclusions}

This study is the first to report comprehensively on the short-term mortality rates of adult patients with hematologic malignancies admitted to the ICU and meeting Sepsis-3 criteria for septic shock. Despite the survival improvements in patients with cancer and sepsis overall reported in the literature over the past 2 decades, as well as the impact of the Sepsis-3 definition, septic shock in patients with hematologic malignancies is still associated with high mortality rates and poor 90-day survival. These results are an urgent call to action with higher awareness, including the further evaluation of interventions such as earlier ICU admission, aminoglycosides administration, and G-CSF treatment.

\section{Acknowledgments}

This manuscript was edited by Sarah Bronson, ELS, of the Research Medical Library at The University of Texas MD Anderson Cancer Center.

Submitted January 27, 2021; final revision received April 13, 2021; accepted for publication April 15, 2021

Previous presentation: Preliminary data used in this study were presented as an abstract at the Society of Critical Care Medicine (SSCM) 49th Critical Care Congress; February 16-19, 2020; Orlando, Florida, and the SSCM 50th Critical Care Virtual Congress; January 31-February 12, 2021. Abstracts 614, 848,886 , and 1208

Author contributions: Study concept and design: Manjappachar, Cuenca, Ramírez, Hernandez, Nates. Data integrity and analysis: Manjappachar Cuenca, Ramírez, Hernandez, Nates. Data acquisition: Manjappachar, Cuenca, Ramírez. Data analysis: Manjappachar, Cuenca, Ramírez. Data interpretation: All authors. Formal statistical analysis: Cuenca, Hernandez, Nates. Administrative, technical, or material support: Cuenca, Hernandez, Martin, Reyes, Heatter, Price, Nates. Manuscript preparation: Manjappachar, Cuenca, Hernandez, Nates. Critical revision: All authors.

Disclosures: Dr. Rathi has reported serving on a data safety monitoring board for Cellenkos Inc. The remaining authors have not received any financial consideration from any person or organization to support the preparation, analysis, results, or discussion of this article.

Funding: Research reported in this publication was supported by the George Sweeney Fellowship, The University of Texas MD Anderson Cance Center Grant Resources, and the $\mathrm{NCl}$ of the $\mathrm{NIH}$ (number P30CA016672; J.L. Nates). 
Disclaimer: The content is solely the responsibility of the authors and does not necessarily represent the official views of the $\mathrm{NIH}$. None of the funders had any role in the conduct of the study; in the collection, management, analysis, or interpretation of the data; in the preparation, review, or approval of the manuscript; or in the decision to submit the manuscript for publication.
Correspondence: Joseph L. Nates, MD, MBA, CMQ, MCCM, Department of Critical Care, Division of Anesthesiology, Critical Care, and Pain Medicine, The University of Texas MD Anderson Cancer Center, 1515 Holcombe Boulevard, Unit \#112, Houston, TX 77030.

Email: jlnates@mdanderson.org

\section{References}

1. National Cancer Institute. Cancer Statistics. Accessed September 30, 2020. Available at: https://www.cancer.gov/about-cancer/understanding/statistics

2. Roser M, Ritchie H, Ortiz-Ospina E. World Population Growth. Our World in Data 2019. Accessed September 30, 2020. Available at: https:// ourworldindata.org/world-population-growth

3. Rozhok Al, DeGregori J. The evolution of lifespan and age-dependent cancer risk. Trends Cancer 2016;2:552-560.

4. Williams MD, Braun LA, Cooper LM, et al. Hospitalized cancer patients with severe sepsis: analysis of incidence, mortality, and associated costs of care. Crit Care 2004;8:R291-298.

5. Lemiale V, Pons S, Mirouse A, et al. Sepsis and septic shock in patients with malignancies: a Groupe de Recherche Respiratoire en Réanimation OncoHématologique study. Crit Care Med 2020;48:822-829.

6. Rhee C, Dantes R, Epstein L, et al. Incidence and trends of sepsis in US hospitals using clinical vs claims data, 2009-2014. JAMA 2017;318:1241-1249.

7. Rudd KE, Johnson SC, Agesa KM, et al. Global, regional, and national sepsis incidence and mortality, 1990-2017: analysis for the Global Burden of Disease Study. Lancet 2020;395:200-211.

8. Liu V, Escobar GJ, Greene JD, et al. Hospital deaths in patients with sepsis from 2 independent cohorts. JAMA 2014;312:90-92

9. Paoli CJ, Reynolds MA, Sinha M, et al. Epidemiology and costs of sepsis in the United States-an analysis based on timing of diagnosis and severity level. Crit Care Med 2018;46:1889-1897.

10. Fleischmann C, Scherag A, Adhikari NKJ, et al. Assessment of global incidence and mortality of hospital-treated sepsis. current estimates and limitations. Am J Respir Crit Care Med 2016;193:259-272.

11. Azoulay E, Mokart D, Pène F, et al. Outcomes of critically ill patients with hematologic malignancies: prospective multicenter data from France and Belgium-a Groupe de Recherche Respiratoire en Réanimation OncoHématologique study. J Clin Oncol 2013;31:2810-2818.

12. Cheng $Q$, Tang $Y, Y$ ang $Q$, et al. The prognostic factors for patients with hematological malignancies admitted to the intensive care unit. Springerplus 2016;5:2038.

13. Singer M, Deutschman CS, Seymour CW, et al. The Third International Consensus Definitions for Sepsis and Septic Shock (Sepsis-3). JAMA 2016; 315:801-810.

14. Zuber B, Tran TC, Aegerter $P$, et al. Impact of case volume on survival of septic shock in patients with malignancies. Crit Care Med 2012;40:55-62.

15. Jeddi R, Ghédira H, Ben Amor R, et al. Risk factors of septic shock in patients with hematologic malignancies and Pseudomonas infections. Hematology 2011;16:160-165.

16. Ñamendys-Silva SA, González-Herrera MO, Texcocano-Becerra J, et al. Clinical characteristics and outcomes of critically ill cancer patients with septic shock. QJM 2011;104:505-511.

17. Shankar-Hari M, Phillips GS, Levy ML, et al. Developing a new definition and assessing new clinical criteria for septic shock: for the Third International Consensus Definitions for Sepsis and Septic Shock (Sepsis-3). JAMA 2016;315:775-787.

18. Nates JL, Nunnally M, Kleinpell R, et al. ICU admission, discharge, and triage guidelines: a framework to enhance clinical operations, development of institutional policies, and further research. Crit Care Med 2016;44:1553-1602.

19. Wallace SK, Rathi NK, Waller DK, et al. Two decades of ICU utilization and hospital outcomes in a comprehensive cancer center. Crit Care Med 2016; 44:926-933.

20. Equator Network. Enhancing the QUAlity and Transparency Of health Research. The Strengthening the Reporting of Observational Studies in Epidemiology (STROBE) Statement: guidelines for reporting observational studies. Accessed September 30, 2020. Available at: https://www.equatornetwork.org/reporting-guidelines/strobe/
21. Hosmer DW, Lemeshow S, Sturdivant RX. Applied Logistic Regression, 3rd ed. Hoboken, NJ: John Wiley \& Sons; 2013

22. Forthofer RN, Lee ES, Hernandez M. Biostatistics: A Guide to Design, Analysis, and Discovery, 2nd ed. Burlington, MA: Elsevier Academic Press; 2006.

23. Society of Critical Care Medicine. Surviving Sepsis Campaign: History. Accessed September 30, 2020. Available at: https://www.sccm.org/ SurvivingSepsisCampaign/About-SSC/History

24. Sprung $C L$, Schein RMH, Balk RA. To SIRS with love-an open letter. Crit Care Med 2017;45:736-738.

25. Sprung $\mathrm{CL}$, Schein RMH, Balk RA. The new sepsis consensus definitions: the good, the bad and the ugly. Intensive Care Med 2016;42:2024-2026.

26. Sterling SA, Puskarich MA, Glass AF, et al. The impact of the Sepsis-3 septic shock definition on previously defined septic shock patients. Crit Care Med 2017;45:1436-1442.

27. Sprung CL, Trahtemberg U. What definition should we use for sepsis and septic shock? Crit Care Med 2017;45:1564-1567

28. Nathan N, Sculier JP, Ameye L, et al. Sepsis and septic shock definitions in patients with cancer admitted in ICU. J Intensive Care Med 2021;36: 255-261. doi:

29. Cooper AJ, Keller SP, Chan C, et al. Improvements in Sepsisassociated mortality in hospitalized patients with cancer versus those without cancer. A 12-year analysis using clinical data. Ann Am Thorac Soc 2020;17:466-473.

30. Hensley MK, Donnelly JP, Carlton EF, et al. Epidemiology and outcomes of cancer-related versus non-cancer-related sepsis hospitalizations. Crit Care Med 2019;47:1310-1316.

31. Kaukonen K-M, Bailey M, Suzuki S, et al. Mortality related to severe sepsis and septic shock among critically ill patients in Australia and New Zealand, 2000-2012. JAMA 2014;311:1308-1316.

32. Vincent JL, Jones G, David S, et al. Frequency and mortality of septic shock in Europe and North America: a systematic review and meta-analysis. Crit Care 2019;23:196.

33. Kumar G, Ahmad S, Taneja A, et al. Severe sepsis in hematopoietic stem cell transplant recipients. Crit Care Med 2015;43:411-421.

34. Bayraktar UD, Shpall EJ, Liu P, et al. Hematopoietic cell transplantation-specific comorbidity index predicts inpatient mortality and survival in patients who received allogeneic transplantation admitted to the intensive care unit. J Clin Oncol 2013;31:4207-4214.

35. Malik IA, Cardenas-Turanzas M, Gaeta S, et al. Sepsis and acute myeloid leukemia: a population-level study of comparative outcomes of patients discharged from Texas hospitals. Clin Lymphoma Myeloma Leuk 2017;17:e27-32.

36. Lahoti A, Kantarjian H, Salahudeen AK, et al. Predictors and outcome of acute kidney injury in patients with acute myelogenous leukemia or high-risk myelodysplastic syndrome. Cancer 2010;116:4063-4068.

37. Georges Q, Azoulay E, Mokart D, et al. Influence of neutropenia on mortality of critically ill cancer patients: results of a meta-analysis on individual data. Crit Care 2018;22:326

38. Legrand M, Max A, Peigne $V$, et al. Survival in neutropenic patients with severe sepsis or septic shock. Crit Care Med 2012;40:43-49.

39. Kumar A, Roberts D, Wood KE, et al. Duration of hypotension before initiation of effective antimicrobial therapy is the critical determinant of survival in human septic shock. Crit Care Med 2006;34:1589-1596.

40. Singer M. Antibiotics for sepsis: does each hour really count, or is it incestuous amplification? Am J Respir Crit Care Med 2017;196:800-802.

41. Goldman JD, Gallaher A, Jain R, et al. Infusion-compatible antibiotic formulations for rapid administration to improve outcomes in cancer outpatients with severe sepsis and septic shock: the Sepsis STAT Pack. J Natl Compr Canc Netw 2017;15:457-464. 
Supplemental online content for:

\section{Outcomes and Predictors of 28-Day Mortality in Patients With Hematologic Malignancies and Septic Shock Defined by Sepsis-3 Criteria}

Nirmala K. Manjappachar, MD; John A. Cuenca, MD; Claudia M. Ramírez, MD; Mike Hernandez, MS; Peyton Martin, BS; Maria P. Reyes, MD; Alba J. Heatter, MD; Cristina Gutierrez, MD; Nisha Rathi, MD; Charles L. Sprung, MD; Kristen J. Price, MD; and Joseph L. Nates, MD, MBA, CMQ, MCCM

J Natl Compr Canc Netw 2022;20(1):45-53

eFigure 1: Flowchart of Included Participants by Hematologic Malignancies

eFigure 2: Patient Distribution Based on SOFA Score and Survival Status at 28 Days

eFigure 3: Risk Stratification Plot

eTable 1: Comorbidities

eTable 2: Laboratory Parameters

eTable 3: Microbiologic Isolates

eTable 4: Interventions

eTable 5: Mortality Rates in Patients With Hematologic Malignancies by Septic Shock Definition

eTable 6: Advance Care Planning

eTable 7: Mortality by Neutropenia Status

eTable 8: Study Cohort by G-CSF Administration 


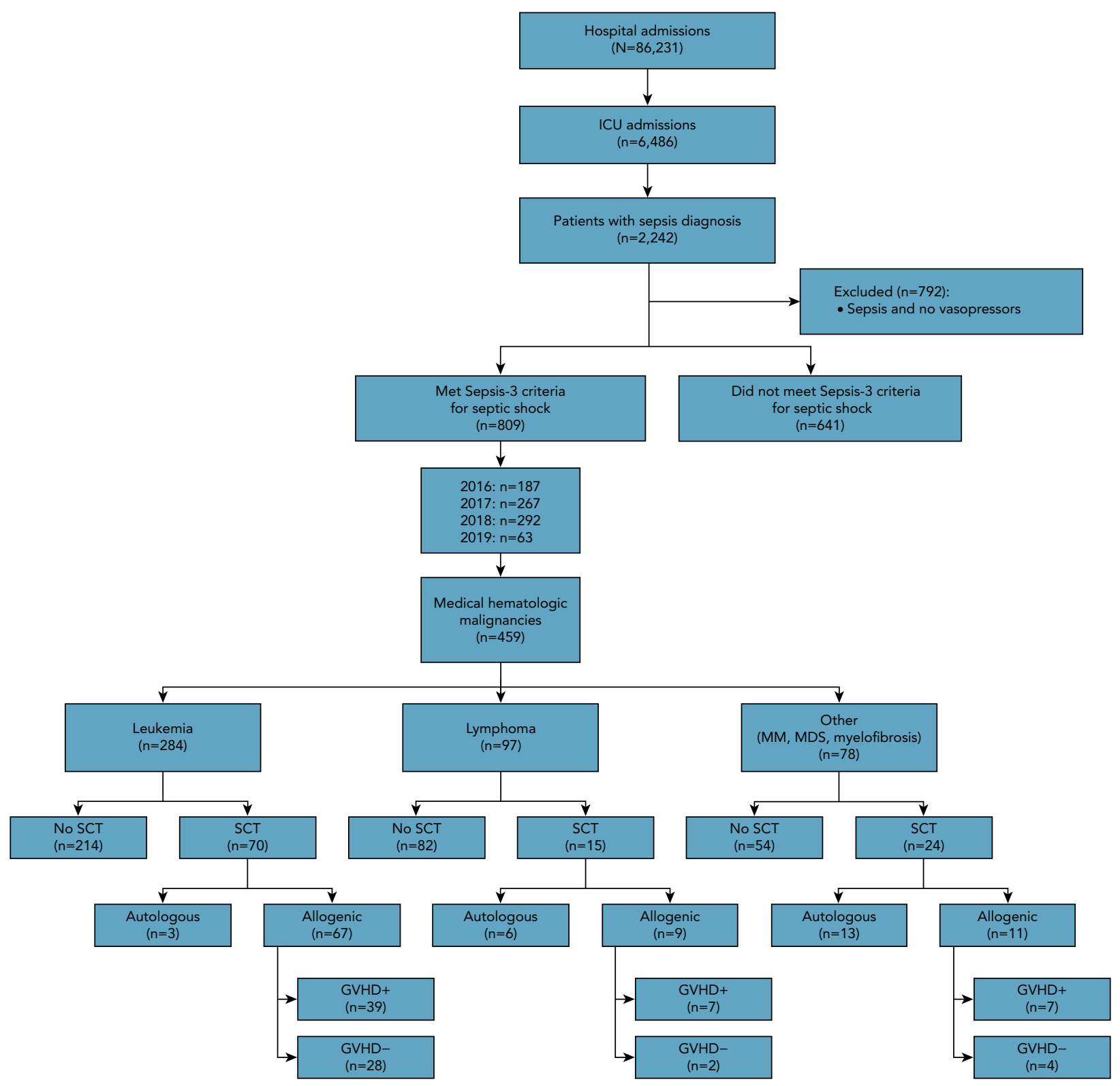

eFigure 1. Flowchart of included participants by hematologic malignancies.

Abbreviations: GVHD, graft-versus-host disease; MDS, myelodysplastic syndromes; MM, multiple myeloma; SCT, stem cell transplant. 


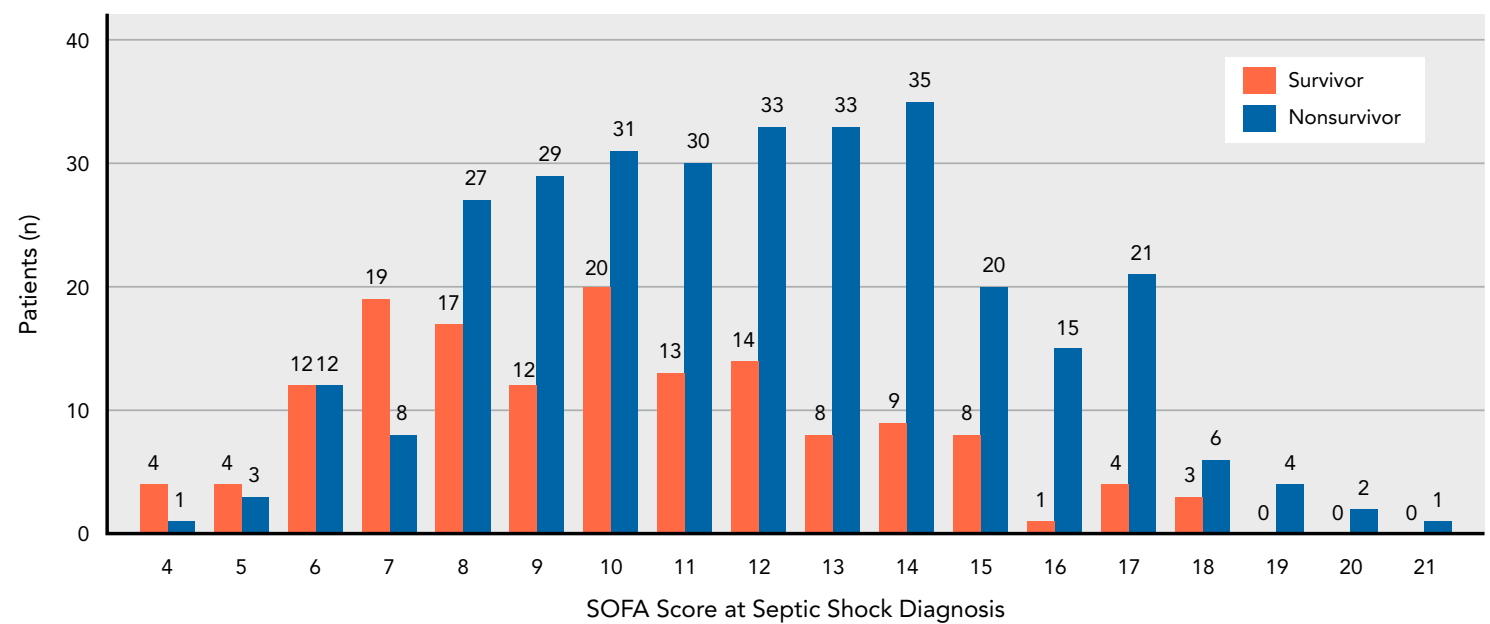

eFigure 2. Patient distribution based on SOFA score and survival status at 28 days.

Abbreviation: SOFA, sequential organ failure assessment score. 


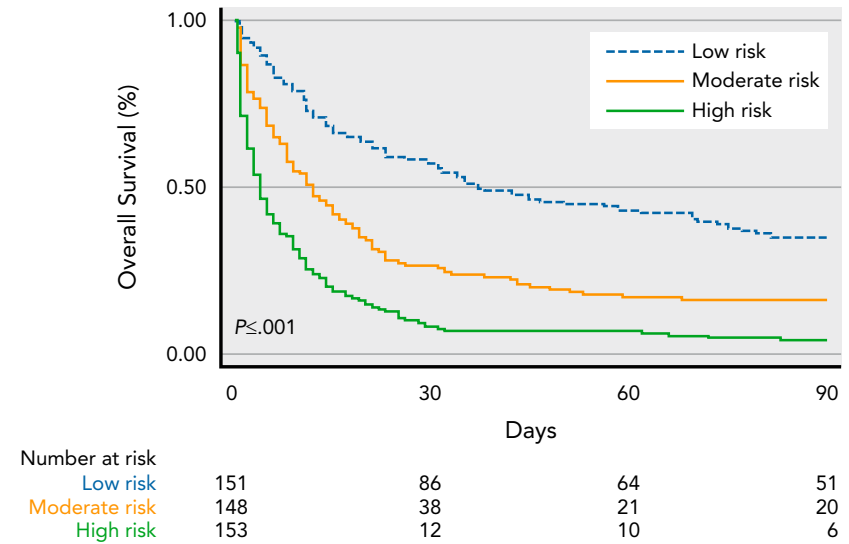

eFigure 3. Risk stratification plot.

This plot illustrates a clear separation of survival distributions based on risk groups derived using the tertiles of the estimated probability of 28-day mortality emanating from the 6-covariate multivariable model developed in our study. 


\section{eTable 1. Comorbidities}

\begin{tabular}{|lllll|}
\hline Comorbidity & $\begin{array}{l}\text { Overall } \\
\mathbf{n}(\%)\end{array}$ & $\begin{array}{l}\text { Survivors } \\
\mathbf{n}(\%)\end{array}$ & $\begin{array}{l}\text { Nonsurvivors } \\
\mathbf{n}(\%)\end{array}$ & 311 \\
\hline Total, $\mathrm{n}$ & 459 & 148 & $28(9)$ & $\mathbf{P}$ Value \\
\hline Myocardial infarction & $38(8.2)$ & $10(6.7)$ & $38(12.2)$ & .414 \\
\hline Congestive heart failure & $56(12.2)$ & $18(12.1)$ & $9(2.8)$ & .986 \\
\hline Peripheral vascular disease & $11(2.3)$ & $2(1.3)$ & $19(6.1)$ & .312 \\
\hline Cerebrovascular disease & $26(5.6)$ & $7(4.7)$ & $1(0.3)$ & .550 \\
\hline Dementia & $2(0.4)$ & $1(0.6)$ & $24(7.7)$ & .590 \\
\hline Chronic pulmonary disease & $34(7.4)$ & $10(6.7)$ & $5(1.6)$ & $6(1.9)$ \\
\hline Connective tissue disease & $8(1.7)$ & $3(2)$ & $92(29.5)$ \\
\hline Peptic ulcer disease & $10(2.1)$ & $144(97.2)$ & $41(13.1)$ \\
\hline Diabetes mellitus & $142(31.0)$ & $98(66.2)$ & $1(0.3)$ \\
\hline Moderate/Severe CKD & $58(12.6)$ & $131(88.5)$ & $15(4.8)$ \\
\hline Hemiplegia & $2(0.4)$ & $147(99.3)$ & $3(0.9)$ & .748 \\
\hline Liver disease & $22(4.7)$ & $7(4.7)$ & $152(48.8)$ \\
\hline AlDS & $5(1)$ & $2(1.3)$ & $59(57.4)$ & $59(18.9)$ \\
\hline Immunosuppression & $237(51.6)$ & $37(25)$ & .363 \\
\hline Steroid therapy & $96(20.9)$ & .609 \\
\hline
\end{tabular}

Categorical variables differences were calculated with chi-square test. Continuous data differences were calculated with $t$ test.

Abbreviation: CKD, chronic kidney disease. 


\section{eTable 2. Laboratory Parameters}

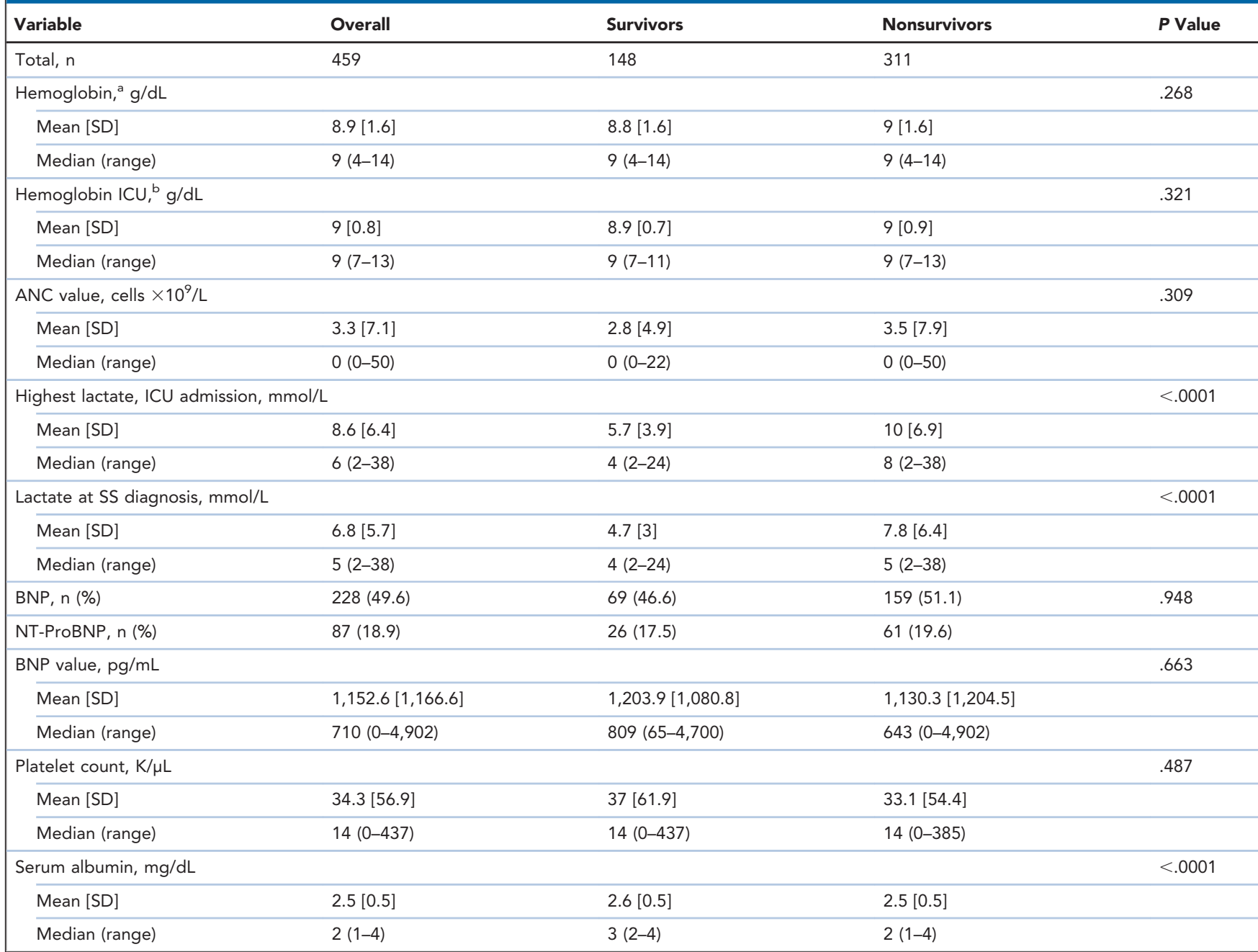

Categorical variables differences were calculated with chi-square test. Continuous data differences were calculated with $t$ test.

Abbreviations: ANC, absolute neutrophil count; BNP, B-type natriuretic peptide; ICU, intensive care unit; NT-proBNP, N-terminal pro-B-type natriuretic peptide;

SS, septic shock.

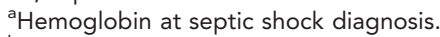

${ }^{\text {b}}$ Mean hemoglobin during the ICU stay. 
6-Manjappachar et al

\section{eTable 3. Microbiologic Isolates}

\begin{tabular}{|c|c|c|c|c|}
\hline Isolate & $\begin{array}{l}\text { Overall } \\
\text { n (\%) }\end{array}$ & $\begin{array}{l}\text { Survivors } \\
\text { n (\%) }\end{array}$ & $\begin{array}{l}\text { Nonsurvivors } \\
\text { n (\%) }\end{array}$ & $P$ Value \\
\hline Total, n & 459 & 148 & 311 & \\
\hline Microbial organism isolated & $306(66.6)$ & $108(72.9)$ & $198(63.6)$ & .048 \\
\hline Gram-positive & $111(24.1)$ & $38(25.6)$ & $73(23.4)$ & .606 \\
\hline Gram-negative & $163(35.5)$ & $63(42.5)$ & $100(32.1)$ & .029 \\
\hline Anaerobes & $12(2.6)$ & $7(4.7)$ & $5(1.6)$ & .05 \\
\hline Fungi & 55 (11.9) & $15(10.1)$ & $40(12.8)$ & .4 \\
\hline Virus & $52(11.3)$ & $22(14.8)$ & $30(9.6)$ & .099 \\
\hline
\end{tabular}

Categorical variables differences were calculated with chi-square test. Continuous data differences were calculated with $t$ test. 


\section{eTable 4. Interventions}

\begin{tabular}{|c|c|c|c|c|}
\hline Intervention & $\begin{array}{l}\text { Overall } \\
\text { n (\%) }\end{array}$ & $\begin{array}{l}\text { Survivors } \\
\text { n (\%) }\end{array}$ & $\begin{array}{l}\text { Nonsurvivors } \\
\text { n (\%) }\end{array}$ & $P$ Value \\
\hline Total, n & 459 & 148 & 311 & \\
\hline \multicolumn{5}{|l|}{ Antibiotics } \\
\hline Aminoglycosides & $281(61.2)$ & $110(74.3)$ & $171(54.9)$ & $<.0001$ \\
\hline Vancomycin & $186(40.5)$ & $69(46.6)$ & $117(37.6)$ & .066 \\
\hline Piperacillin-tazobactam & $134(29.1)$ & $49(33.1)$ & $85(27.3)$ & .203 \\
\hline Linezolid & 309 (67.3) & $102(68.9)$ & 207 (66.5) & .614 \\
\hline Meropenem & $343(74.7)$ & $126(85.1)$ & $217(69.7)$ & $<.0001$ \\
\hline Metronidazole & $109(23.7)$ & $36(24.3)$ & $73(23.4)$ & .841 \\
\hline Daptomycin & $119(25.9)$ & $46(31)$ & $73(23.4)$ & .082 \\
\hline Cefepime & $200(43.5)$ & $83(56)$ & $117(37.6)$ & $<.0001$ \\
\hline Aztreonam & $21(4.5)$ & $8(5.4)$ & $13(4.1)$ & .557 \\
\hline Clindamycin & $27(5.8)$ & $9(6)$ & $18(5.7)$ & .901 \\
\hline Minocycline & $66(14.3)$ & $17(11.4)$ & $49(15.7)$ & .223 \\
\hline Ceftazidime & $118(25.7)$ & $28(18.9)$ & $90(28.9)$ & .022 \\
\hline Tigecycline & $134(29.1)$ & $39(26.3)$ & $95(30.5)$ & .355 \\
\hline Ciprofloxacin & $33(7.1)$ & $19(12.8)$ & $14(4.5)$ & .001 \\
\hline Sulfamethoxazole & 139 (30.2) & $43(29)$ & $96(30.8)$ & .693 \\
\hline \multicolumn{5}{|l|}{ Vasoactive agents } \\
\hline Norepinephrine & 453 (98.6) & $143(96.6)$ & $310(99.6)$ & .007 \\
\hline Epinephrine & $111(24.1)$ & $35(23.6)$ & $76(24.4)$ & .854 \\
\hline Vasopressin & $258(56.2)$ & $63(42.5)$ & $195(62.7)$ & $<.0001$ \\
\hline Angiotensin II & $3(0.6)$ & $1(0.6)$ & $2(0.6)$ & .968 \\
\hline Phenylephrine & $92(20)$ & $17(11.4)$ & $75(24.1)$ & .002 \\
\hline Dobutamine & $2(0.4)$ & $1(0.6)$ & $1(0.3)$ & .59 \\
\hline \multicolumn{5}{|l|}{ Steroids } \\
\hline Any steroid & $414(90.1)$ & $137(92.5)$ & 277 (89) & .239 \\
\hline Hydrocortisone & $383(83.4)$ & $131(88.5)$ & $252(81)$ & .044 \\
\hline Methylprednisolone & $185(40.3)$ & $58(39.1)$ & $127(40.8)$ & .737 \\
\hline Prednisolone & $14(3)$ & $8(5.4)$ & $6(1.9)$ & .043 \\
\hline Dexamethasone & $48(10.4)$ & $16(10.8)$ & $32(10.2)$ & .865 \\
\hline Fludrocortisone & $14(3)$ & $7(4.7)$ & $7(2.2)$ & .149 \\
\hline \multicolumn{5}{|l|}{ Other } \\
\hline Thiamine & $228(49.6)$ & $75(50.6)$ & $153(49.1)$ & .767 \\
\hline Vitamin C & $44(9.5)$ & $16(10.8)$ & $28(9)$ & .539 \\
\hline Antiviral & $281(61.2)$ & $92(62.1)$ & 189 (60.7) & .775 \\
\hline Antifungals & $375(81.6)$ & 118 (79.7) & $257(82.6)$ & .452 \\
\hline G-CSF & $125(27.2)$ & $55(37.1)$ & $70(22.5)$ & .001 \\
\hline
\end{tabular}

Categorical variables differences were calculated with chi-square test. Continuous data differences were calculated with $t$ test.

Abbreviation: G-CSF, granulocyte colony-stimulating factor 
eTable 5. Mortality Rates in Patients With Hematologic Malignancies by Septic Shock Definition

\begin{tabular}{|c|c|c|c|c|}
\hline Outcome & $\begin{array}{l}\text { Sepsis + Vasopressors } \\
\text { n (\%) }\end{array}$ & $\begin{array}{l}\text { Did Not Meet } \\
\text { Sepsis-3 Criteria } \\
\text { for Septic Shock } \\
\text { n (\%) }\end{array}$ & $\begin{array}{l}\text { Met } \\
\text { Sepsis-3 Criteria } \\
\text { for Septic Shock } \\
\text { n (\%) }\end{array}$ & $P$ Value \\
\hline ICU mortality & $419(50.7)$ & $126(34.3)$ & $293(63.8)$ & $<.0001$ \\
\hline Hospital mortality & $504(61)$ & $165(44.9)$ & 339 (73.9) & $<.0001$ \\
\hline
\end{tabular}

Categorical variables differences were calculated with chi-square test.

\begin{tabular}{|lllll|}
\hline eTable 6. Advance Care Planning & & & \\
\hline Point of Death & $\begin{array}{l}\text { Full Code + DNR } \\
\mathbf{n}\end{array}$ & $\begin{array}{l}\text { Full Code at } \\
\text { Time of Death } \\
\mathbf{n}(\%)\end{array}$ & $\begin{array}{l}\text { DNR at } \\
\text { Time of Death } \\
\mathbf{n}(\%)\end{array}$ & $\begin{array}{l}\text { Comfort Care } \\
\mathbf{n}(\%)\end{array}$ \\
\hline ICU discharge & 293 & $22(7.5)$ & $271(92.4)$ & $191(65.1)$ \\
\hline Hospital discharge & 339 & $42(12.3)$ & $297(87.6)$ & $209(61.6)$ \\
\hline 28 days & 311 & $40(12.8)$ & $271(87.1)$ & $192(61.7)$ \\
\hline 90 days & 370 & $68(18.3)$ & $302(81.6)$ & $209(56.4)$ \\
\hline
\end{tabular}

Abbreviations: DNR, do-not-resuscitate order; ICU, intensive care unit.

\section{eTable 7. Mortality by Neutropenia Status}

\begin{tabular}{|llll|}
\hline Variable & $\begin{array}{l}\text { Severe Neutropenia } \\
\mathbf{n}(\%)\end{array}$ & $\begin{array}{l}\text { No Neutropenia } \\
\mathbf{n}(\%)\end{array}$ & $\boldsymbol{P ~ V a l u e ~}^{\mathbf{b}}$ \\
\hline Total, $\mathrm{n}$ & 247 & 208 & $<.0001$ \\
\hline G-CSF & $90(36.4)$ & $34(16.3)$ & .712 \\
\hline ICU mortality & $155(62.8)$ & $134(64.4)$ & .143 \\
\hline Hospital mortality & $175(70.9)$ & $160(76.9)$ & .520 \\
\hline 28-day mortality & $164(66.4)$ & $144(69.2)$ & .068 \\
\hline 90-day mortality & $191(77.3)$ & $175(84.1)$ & \\
\hline
\end{tabular}

Categorical variables differences were calculated with chi-square test.

Abbreviations: ANC, absolute neutrophil count; G-CSF: granulocyte colony-stimulating factor; ICU, intensive care unit.

${ }^{a} \mathrm{ANC} \leq 500 / \mathrm{mm}^{3}$.

${ }^{\mathrm{b}} \mathrm{ANC}>500 / \mathrm{mm}^{3}$. 


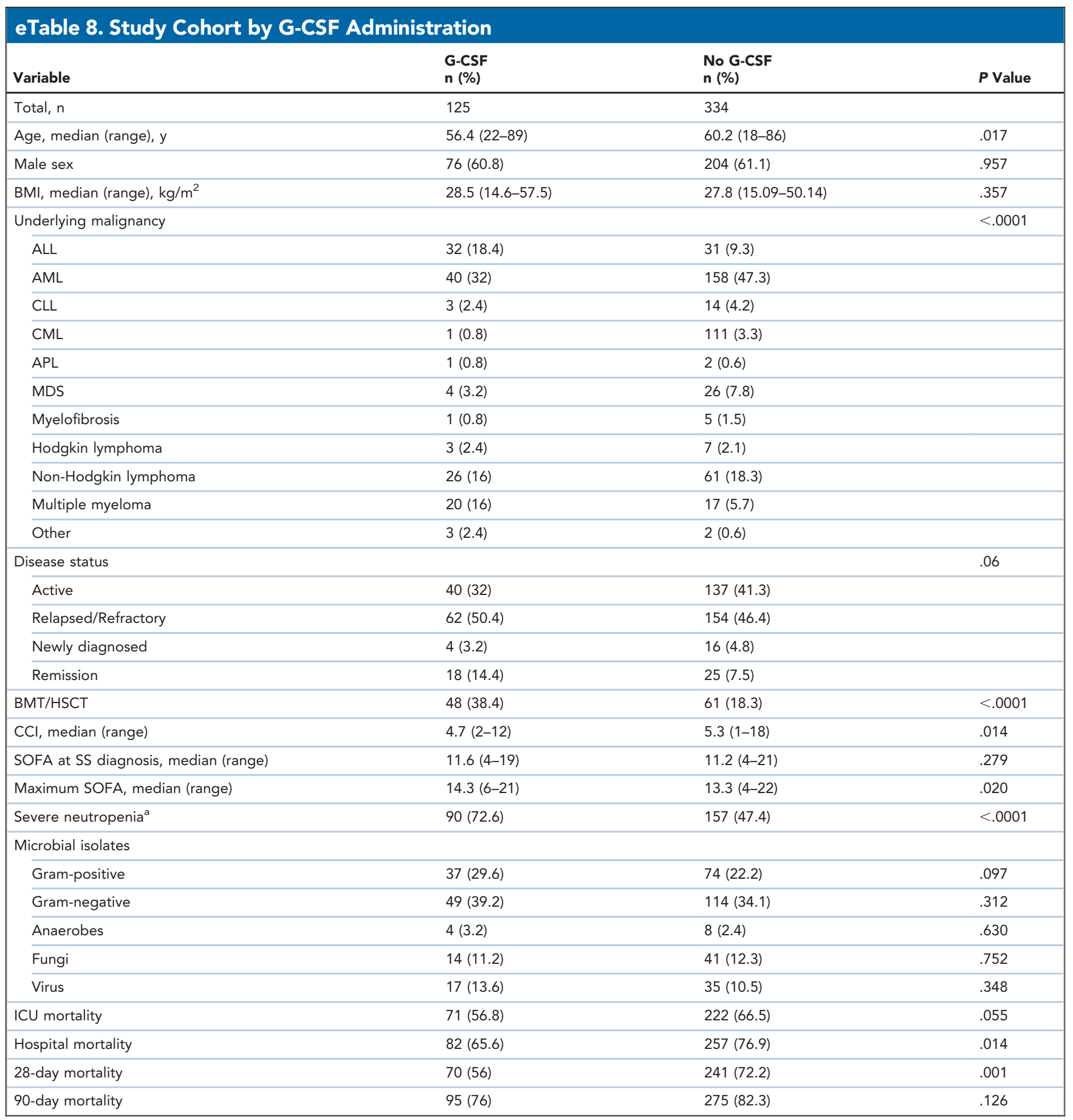

Abbreviations: ALL, acute lymphocytic leukemia; AML, acute myeloid leukemia; APL, acute promyelocytic leukemia; BMI, body mass index; BMT, bone marrow transplant; $\mathrm{CCl}$, Charlson comorbidity index; CLL, chronic lymphocytic leukemia; CML, chronic myeloid leukemia; G-CSF, granulocyte colony-stimulating factor; HSCT, hematopoietic stem cell transplant; ICU, intensive care unit; MDS, myelodysplastic syndromes; SOFA, sequential organ failure assessment score; SS, septic shock.

${ }^{a}$ Absolute neutrophil count $<500 / \mathrm{mm}^{3}$. 